\title{
Exactitud de la punción aspiración con aguja fina en el diagnóstico de cáncer diferenciado de tiroides
}

\section{Diagnostic accuracy of fine needle aspiration puncture for diagnosing differentiated thyroid cancer}

\author{
María C. Pereyra ${ }^{1}$, Romina A. Gecchelin ${ }^{1}$, Mauro J. Pautasso ${ }^{1}$, Constanza F Ramacciotti ${ }^{1}$, Analía V. \\ Checa $^{1}$, María L. Bertolino ${ }^{1}$, Eduardo N. Cohen ${ }^{1}$ \\ 1Universidad católica de Córdoba. Clínica Universitaria Reina Fabiola. Servicio de Endocrinología. \\ Correspondencia: Pereyra María Celina. Servicio de Endocrinología Clínica Universitaria Reina Fabiola Oncativo 1248-X5004FHP-Cordoba, Argentina; email: \\ mariacelinapereyra@gmail.com.
}

\section{Resumen}

INTRODUCCIÓN: La importancia clínica de los nódulos tiroideos (NT) radica en la necesidad de excluir cáncer de tiroides ( 7 al 15\% de los casos). En Argentina representa el 2,2\% y 0,5\% de los cánceres que ocurren anualmente en mujeres y hombres respectivamente. La citología por punción aspiración con aguja fina (PAAF) de NT estima el riesgo de malignidad y es la prueba confirmatoria de elección para el diagnóstico, con una especificidad reportada del $94 \%$.

OBJETIVO: Determinar la exactitud diagnóstica de los criterios citopatológicos del Sistema Bethesda (SB) obtenidos por PAAF de NT para el diagnóstico de cáncer de tiroides. Establecer la frecuencia de cada categoría del SB en nuestra población. Calcular el porcentaje de malignidad para cada categoría del SB en nuestra población.

PACIENTES Y MÉTODOS: Estudio observacional, retrospectivo, de pruebas diagnósticas. Se incluyeron pacientes adultos con NT sospechosos de malignidad por ecografía y PAAF. Se analizaron las historias clínicas de pacientes de ambos sexos intervenidos mediante cirugía tiroidea entre el 1 de enero de 2012 y el 31 de diciembre de 2018 en la Clínica Universitaria Reina Fabiola de la Ciudad de Córdoba. Se evaluó la sensibilidad, especificidad, valores predictivos positivo y negativo de los criterios citopatológicos del SB obtenidos por PAAF de NT tomando como estándar de oro el resultado anatomopatológico.

RESULTADOS: Se incluyeron 191 pacientes. De ellos el 82,20\% eran de sexo femenino y la media de edad fue 43,45 $\pm 13,29$. Presentaron diagnóstico de cáncer de tiroides 89 pacientes, correspondiendo la totalidad a CDT. Las tasas de malignidad para las categorías del SB II, III, IV y V fueron del 18,5; 31,6; 62,8 y $90,5 \%$, respectivamente. Al analizar la utilidad del SB como prueba de screening encontramos una sensibilidad para detectar malignidad del $84,6 \%$, con una especificidad del 72,6\%, un VPP del 76,7\%, un VPN del 81,5\% y una exactitud diagnóstica global del 78,8\%. Este análisis, en punciones altamente sospechosas, aumentó la exactitud diagnóstica de la prueba hasta el 85,2\%, sensibilidad 76,5\%, especificidad $93 \%$, VPP 90,7\% y VPN $81,5 \%$.

CONCLUSIONES: La exactitud diagnóstica de los criterios citopatológicos del Sistema Bethesda (SB) obtenidos por PAAF de nódulos tiroideos para el diagnóstico de cáncer de tiroides es buena, por lo cual es uno de los métodos recomendados en el algoritmo diagnóstico de cáncer de tiroides.

Palabras claves: Cáncer de tiroides, biopsia con aguja fina, nódulo tiroideo. 


\section{Abstract}

INTRODUCTION: The importance of thyroid nodules (NT) lies in the need to exclude thyroid cancer, which occurs in 7 to $15 \%$ of cases. In Argentina it represents $2.2 \%$ and $0.5 \%$ of all cancers that occur annually in women and men respectively. NT fine needle aspiration (PAAF) cytology estimates the risk of malignancy and is the confirmatory test for diagnosis, with a specificity of $94 \%$.

OBJECTIVE: To determine the diagnostic accuracy of the cytopathological criteria of the BS obtained by TN FNA for the diagnosis of thyroid cancer. To establish the frequency of each BS category in our population. To alculate the percentage of malignancy for each category of the BS in our population.

PATIENTS AND METHODS: Observational, retrospective, diagnostic test study in which adult patients with ultrasound suspicious for malignancy TN and FNA were included. Data of patients who underwent thyroid surgery between January 1, 2012 and December 31, 2018 at the Reina Fabiola University Clinic in Córdoba City were analyzed. Sensitivity, specificity, positive and negative predictive values of the cytopathological criteria of the BS obtained by FNA were evaluated, taking as a gold standard the pathological result.

RESULTS: 191 patients were included. $82.20 \%$ were female and the mean age was $43.45 \pm 13.29$. 89 patients were diagnosed of thyroid cancer, all of them classified as differentiated thyroid carcinoma (DTC). Regarding the percentages of malignancy in the different categories of the Bethesda system, the rates of malignancy for categories II, III, IV and V were $18.5 \% ; 31.6 \% ; 62.8 \%$ and $90.5 \%$, respectively. Regarding the performance of the Bethesda system, when analyzing its usefulness as a screening test (analysis 1: category II vs. IV + V + VI) we found a sensitivity to detect malignancy of $84.6 \%$, with a specificity of $72.6 \%$, a PPV of $76.7 \%$, a NPV of $81.5 \%$ and a global diagnostic accuracy of $78.8 \%$. This analysis, in highly suspicious punctures (analysis 2: category II vs. V + VI), increased the diagnostic accuracy of the test to $85.2 \%$, sensitivity $76.5 \%$, specificity $93 \%$, PPV $90.7 \%$ and NPV $81.5 \%$.

CONCLUSIONS: The diagnostic accuracy of the BS used for the cytopathological evaluation of the risk of malignancy in the FNA of thyroid nodules is good, which is why it is one of the recommended methods in the diagnostic algorithm of thyroid cancer.

Key words: Thyroid neoplasms, fine-needle aspiration biopsy, thyroid nodules.

\section{Introducción}

Los nódulos tiroideos (NT) palpables se encuentran en el $5 \%$ de mujeres y $1 \%$ de hombres en zonas iodo suficientes ${ }^{1}$. Cuando se utiliza la ecografía como método de screening se pueden detectar en el 19 al $68 \%$ de la población, principalmente mujeres adultas ${ }^{1}$.

La importancia clínica de los NT radica en la necesidad de excluir cáncer de tiroides, que ocurre en el 7 al $15 \%$ de los casos ${ }^{1}$. El cáncer diferenciado de tiroides (CDT) es la neoplasia endócrina maligna más común y constituye el 1-2\% de todos los cánceres. En general, se trata de un tumor con buen pronóstico, con una supervivencia a 5 años de un $98 \%$, y a 10 años superior a un $85 \%{ }^{2}$. El CDT incluye al cáncer papilar $\mathrm{y}$ folicular, que comprenden la gran mayoría (>90\%) de todos los cánceres de tiroides ${ }^{1}$. En la mayoría de casos la enfermedad está confinada a la región cervical en el momento del diagnóstico, con un alto potencial curativo luego de la intervención quirúrgica ${ }^{3,4,5}$.

La incidencia de cáncer de tiroides aumentó exponencialmente en todo el mundo a expensas de pequeños tumores de estirpe papilar ${ }^{3}$. Sin embargo, a pesar del aumento en la incidencia, la mortalidad se mantuvo estable en las últimas tres décadas ${ }^{3}$.

En Argentina, representa el 2,2\% de todos los cánceres que ocurren anualmente en mujeres, ubicando al cáncer de tiroides en el décimo lugar. En los varones, corresponde apenas al $0,5 \%$ del total de casos detectados, ocupando el vigésimo lugar ${ }^{6}$.

La ecografía y la citología por aspiración con aguja fina (PAAF) son las herramientas más utilizadas para la evaluación inicial de $\mathrm{NT}^{1,7,8,9,10}$. La citología por PAAF estima el riesgo de malignidad y es la prueba confirmatoria de elección para el diagnóstico y tratamiento más adecuados de los NT, con una especificidad reportada del $94 \%{ }^{11,12}$. Desde 2007, el Sistema Bethesda (SB) forma parte de la evaluación citopatológica del riesgo de malignidad de la PAAF de los $\mathrm{NT}^{1,7}$. Este sistema reconoce seis categorías diagnósticas y provee una estimación del riesgo de malignidad para cada una de ellas ${ }^{1,7}$. Si bien es una herramienta útil pues permite establecer un mismo idioma para estimar el riesgo de malignidad, se reportaron variaciones principalmente en las categorías indeterminadas (III, IV y V) dependientes del operador, por lo que 
se aconseja que cada centro establezca sus riesgos de malignidad para cada categoría ${ }^{1,13}$.

\section{Objetivo}

Determinar la exactitud diagnóstica de los criterios citopatológicos del Sistema Bethesda (SB) obtenidos por PAAF de NT para el diagnóstico de cáncer de tiroides tomando como estándar de oro el resultado anatomopatológico.

\section{Objetivos secundarios}

- Establecer la frecuencia de cada categoría del SB en nuestra población.

- Calcular el porcentaje de malignidad para cada categoría del SB en nuestra población.

\section{Pacientes y métodos}

Se realizó un estudio observacional, retrospectivo, de pruebas diagnósticas en el que se incluyeron pacientes adultos con NT sospechosos de malignidad por ecografía y punción aspirativa. En estos pacientes se evaluó la sensibilidad, especificidad, valores predictivos positivo (VPP) y negativo (VPN) de los criterios citopatológicos del SB obtenidos por PAAF tomando como estándar de oro el resultado anatomopatológico.

Se analizaron las historias clínicas de pacientes adultos de ambos sexos intervenidos mediante cirugía tiroidea entre el $1^{\circ}$ de enero de 2012 y el 31 de diciembre de 2018 en la Clínica Universitaria Reina Fabiola (CURF) de la Ciudad de Córdoba. Se utilizó el SB de informe citopatológico para clasificación de la PAAF, con sus categorías de recomendación diagnóstica: I: insatisfactorio/no diagnóstico, II: benigno, III: atipias de significado incierto/lesión folicular de significado incierto, IV neoplasia folicular/sospecha de neoplasia folicular, V: sospechoso de malignidad, VI: maligno ${ }^{1}$.

\section{Criterios de exclusión:}

- Pacientes sometidos a tiroidectomía por el Servicio de Cirugía de Cabeza y Cuello que hubiesen sido derivados por servicios de endocrinología externos al de la CURF.

- Pacientes que no contaban con al menos una PAAF tiroidea prequirúrgica.

- Pacientes que fueron sometidos a tiroidectomía por hipertiroidismo.

- Pacientes con diagnóstico anatomopatológico final maligno, tras intervención de tiroidectomía, diferente a CDT.

\section{Variables a consignar:}

1. Edad

2. Sexo

3. Sistema Bethesda de informe citopatológico: Categorías de recomendación diagnóstica ${ }^{1}$.

A. I: insatisfactorio/no diagnóstico

B. II: benigno

C. III: atipias de significado incierto/lesión folicular de significado incierto

D. IV neoplasia folicular/sospecha de neoplasia folicular

E. V: sospechoso de malignidad

F. VI: maligno

4. Anatomía patológica de pieza quirúrgica de tiroidectomía:

A. Maligno: cáncer de tiroides.

B. Benigno ${ }^{14}$ : adenoma folicular, tumor trabecular hialinizante, adenoma de células de Hurtle, tumor de potencial maligno incierto.

5. Tipos de $\mathrm{CDT}^{14}$ :

A. Carcinoma papilar: clásico, microcarcinoma papilar y variantes: encapsulada, folicular, esclerosante difusa, de células altas, células columnares, cribiformemorular, de células en clavo (hobnail), con estroma tipo fibromatosis/fascitis, variante sólida/trabecular, oncocítica, de células fusiformes, células claras y de tipo Warthin.

B. Carcinoma folicular: mínimamente invasivo (encapsulado con invasión capsular solo), encapsulado angioinvasivo y ampliamente invasivo

C. Carcinoma de células de Hurthle

\section{Tamaño muestral}

Para una especificidad de los criterios del SB obtenidos por PAAF para el diagnóstico de cáncer de tiroides del 94\%, un nivel de fiabilidad del $95 \%$ y un error del alfa del 5\%, el tamaño muestral se calculó en 96.

\section{Análisis estadístico}

Los resultados se expresaron como porcentajes para variables categóricas, y como media y desviación estándar para variables continuas, utilizando la mediana y el rango intercuartílico para variables con distribución asimétrica. El análisis estadístico se realizó mediante el programa SPSS1 23.0 para Windows. 


\section{Análisis estadístico de los criterios del SB obtenidos por PAAF de NT}

Se calcularon los parámetros de prueba diagnóstica sensibilidad, especificidad, valores predictivos (valor predictivo positivo [VPP], valor predictivo negativo [VPN]) y exactitud diagnóstica para detectar malignidad mediante dos análisis. En el análisis como prueba de screening (análisis 1) se consideró el resultado de la PAAF como indicación de cirugía por sospecha de malignidad (categorías del BS II vs. IV, V, VI). Según este análisis, los términos "positivo" o "negativo" constituyeron la existencia o no de indicación quirúrgica de cara al análisis estadístico. Se realizó un segundo análisis que midió la capacidad de la prueba para detectar malignidad (análisis 2) ante punciones de alta sospecha (categorías V y VI) frente a pacientes con punción de benignidad (categoría II). La sensibilidad, especificidad, valores predictivos positivo y negativo se obtuvieron con tablas de $2 \times 2$. Las categorías del SB I y III fueron excluidas en los análisis 1 y 2 debido a que sus definiciones son: no diagnóstica (I) y lesión folicular de significado incierto (III).

\section{Recaudos éticos}

Estudio de bajo riesgo: Categoría I de OMS. Según la ley Nacional (ANMAT) Guía de Buenas Prácticas Clínicas en Investigación en Salud y la Ley Provincial 9694 de la provincia de Córdoba, sólo requiere protección de datos. No requirió Consentimiento Informado. La confidencialidad de los datos personales de los pacientes estuvo protegida según la Ley 25.326, registro de datos, artículo 8, resguardando datos personales y secreto profesional.

\section{Resultados}

Se revisaron 395 historias clínicas de pacientes intervenidos de tiroidectomías en la CURF en el periodo establecido. De ellos, se excluyeron 150 pacientes por no disponer de seguimiento clínico, 42 por no tener PAAF tiroidea previa a la cirugía, 10 por haber sido operados por hipertiroidismo, 1 debido a que presentó como diagnóstico final de su tiroidectomía una metástasis de tumor oculto que resultó cáncer de colon y 1 por resultar su pieza quirúrgica de tiroidectomía una histiocitosis tiroidea siendo ésta la primera manifestación de histiocitosis sistémica. Por lo tanto, se incluyeron 191 pacientes. De ellos el $82,20 \%$ eran de sexo femenino y la media de edad fue 43,45 \pm 13,29 años. El adenoma folicular fue el tipo de tumor más frecuente $(86,27 \%)$ dentro de los pacientes con resultado de anatomía patológica benigna (Tabla 1).

Ochenta y nueve pacientes presentaron diagnóstico de cáncer de tiroides, siendo la totalidad de los mismos clasificados como CDT. El $78,65 \%$ eran de sexo femenino y la media de edad fue 39,78 $\pm 12,62$ años. La media del tamaño tumoral fue $20,75 \pm 12,74 \mathrm{~mm}$, el $94,38 \%$ resultaron CDT papilar cuya variante más frecuente fue la clásica con 61 de 84 casos. (Tabla $1)$.

\section{Análisis del Sistema Bethesda}

La categoría diagnóstica del SB más frecuente encontrada en nuestra población fue la II (benigno) con un $34 \%$ de los casos (Tabla 2).

Tabla 1. Descripción anatomopatológica de los tumores tiroideos

\begin{tabular}{lll}
\hline TAMAÑO DE TUMORES TIROIDEOS & Media & DE \\
Tamaño tumoral (mm) & 23,14 & 13,69 \\
& & \\
TAMAÑO DE TUMORES MALIGNOS & Media & DE \\
Tamaño tumoral (mm) & 20,75 & 12,74
\end{tabular}

CARACTERÍSTICAS ANATOMOPATOLÓGICAS TUMORES BENIGNOS

\begin{tabular}{|c|c|c|}
\hline & $\mathrm{N}$ & Porcentaje \\
\hline - Adenoma Folicular & 88 & 86,27 \\
\hline - Adenoma de células de Hurtle & 12 & 11,76 \\
\hline - Tumor trabecular hialinizante & 1 & 0,98 \\
\hline - Tumor de potencial maligno incierto & 1 & 0,98 \\
\hline
\end{tabular}

CARACTERÍSTICAS ANATOMOPATOLÓGICAS TUMORES MALIGNOS

\begin{tabular}{|lll} 
& N & Porcentaje \\
\hline Carcinoma papilar & 84 & 94,38 \\
\hline \multicolumn{1}{|c}{ - Variante clásico } & 61 & 68,54 \\
\hline - Variante folicular & 17 & 19,10 \\
\hline - Variante células altas & 3 & 3,37 \\
\hline - Variante oncocítica & 3 & 3,37 \\
\hline Carcinoma de células de Hurtle & 5 & 5,62 \\
\hline Total de carcinomas tiroideos & 89 & \\
\hline
\end{tabular}


Pereyra MC, Gecchelin RA, Pautasso MJ, Ramacciotti CF, Checa AV, Bertolino ML, Cohen EN. Exactitud de la punción aspiración con aguja fina en el diagnóstico de cáncer diferenciado de tiroides.

Tabla 2. Distribución según categorías diagnósticas del sistema Bethesda

\begin{tabular}{|l|l|l|}
\hline \multirow{2}{*}{ Categoría diagnóstica } & \multicolumn{2}{|l|}{ Total de casos } \\
\cline { 2 - 3 } & Número de casos & Porcentaje \\
\hline I & 21 & $11 \%$ \\
\hline II & 65 & $34 \%$ \\
\hline III & 19 & $10 \%$ \\
\hline IV & 43 & $23 \%$ \\
\hline V & 42 & $22 \%$ \\
\hline VI & 1 & $0,5 \%$ \\
\hline Total & 191 & $100,0 \%$ \\
\hline
\end{tabular}

Referencias: I: insatisfactorio/no diagnóstico, II: benigno, III: atipias de significado incierto/lesión folicular de significado incierto, IV: neoplasia folicular/sospecha de neoplasia folicular, V: sospechoso de malignidad, VI: maligno

Los porcentajes de malignidad en las diferentes categorías del sistema Bethesda se muestran en la Tabla 3. El porcentaje de malignidad fue mayor en las categorías IV y V. La categoría VI contaba con un solo paciente que resultó un CPT.

Tabla 3. Porcentajes de malignidad en las diferentes categorías del sistema Bethesda

\begin{tabular}{|l|l|l|l|l|}
\hline \multirow{2}{*}{ Categoría diagnóstica } & \multicolumn{2}{|l|}{ Maligno } & \multicolumn{2}{l|}{ Benigno } \\
\cline { 2 - 5 } & $\begin{array}{l}\text { Número } \\
\text { de }\end{array}$ & Porcentaje & $\begin{array}{l}\text { Número } \\
\text { de } \\
\text { casos }\end{array}$ & Porcentaje \\
\hline I & 5 & $23,8 \%$ & 16 & $76,2 \%$ \\
\hline II & 12 & $18,5 \%$ & 53 & $81,5 \%$ \\
III & 6 & $31,6 \%$ & 13 & $68,4 \%$ \\
IV & 27 & $62,8 \%$ & 16 & $37,2 \%$ \\
\hline V & 38 & $90,5 \%$ & 4 & $9,5 \%$ \\
\hline Total & 88 & $46,3 \%$ & 102 & $53,7 \%$ \\
\hline
\end{tabular}

Referencias: I: insatisfactorio/no diagnóstico, II: benigno, III: atipias de significado incierto/lesión folicular de significado incierto, IV: neoplasia folicular/sospecha de neoplasia folicular, V: sospechoso de malignidad, VI: maligno.

El rendimiento del sistema Bethesda, al analizar su utilidad como prueba de screening (análisis 1: categoría II vs. IV + V + VI), se describe en la Tabla 4.
Tabla 4. Parámetros de prueba diagnóstica del sistema Bethesda. Análisis 1 (Bethesda II vs IV-V-VI)*

\begin{tabular}{|c|c|c|c|}
\hline \multirow[b]{2}{*}{ Indicadores } & \multirow[b]{2}{*}{$\begin{array}{l}\text { Valo } \\
\text { r }\end{array}$} & \multicolumn{2}{|c|}{$95 \%$ I.C. } \\
\hline & & $\begin{array}{l}\text { Límite } \\
\text { inferior }\end{array}$ & $\begin{array}{l}\text { Límite } \\
\text { superior }\end{array}$ \\
\hline $\begin{array}{ll}\text { Pacientes } & \text { correctamente } \\
\text { diagnosticados } & \end{array}$ & $\begin{array}{l}78,8 \\
\%\end{array}$ & $71,3 \%$ & $84,9 \%$ \\
\hline \multirow{2}{*}{ Sensibilidad } & 84,6 & & \\
\hline & $\%$ & $74,3 \%$ & $91,5 \%$ \\
\hline \multirow{2}{*}{ Especificidad } & 72,6 & & \\
\hline & $\%$ & $60,7 \%$ & $82,1 \%$ \\
\hline \multirow{2}{*}{ Valor predictivo positivo } & 76,7 & & \\
\hline & $\%$ & $66,2 \%$ & $84,9 \%$ \\
\hline \multirow{2}{*}{ Valor predictivo negativo } & 81,5 & & \\
\hline & $\%$ & $69,6 \%$ & $89,7 \%$ \\
\hline
\end{tabular}

II: 65 pacientes, IV-V-VI: 86 pacientes.

*Considera casos en categoría diagnóstica (CD) $I V+V+V I$ verdaderos positivos y casos en CD II verdaderos negativos.

Este análisis, en punciones altamente sospechosas (análisis 2: categoría II vs. V + VI), aumentó la precisión diagnóstica (pacientes correctamente diagnosticados) de la prueba hasta el $85,2 \%$. Los resultados de sensibilidad, especificidad, VPP y VPN se describen en la Tabla 5.

Tabla 5. Parámetros de prueba diagnóstica del sistema Bethesda. Análisis 2 (Bethesda II vs V-VI)*

\begin{tabular}{|c|c|c|c|}
\hline \multirow[b]{2}{*}{ Indicadores } & \multirow[b]{2}{*}{ Valo } & \multicolumn{2}{|c|}{$95 \%$ I.C. } \\
\hline & & $\begin{array}{l}\text { Límite } \\
\text { inferior }\end{array}$ & $\begin{array}{l}\text { Límite } \\
\text { superior }\end{array}$ \\
\hline $\begin{array}{ll}\text { Pacientes } & \text { correctamente } \\
\text { diagnosticados } & \end{array}$ & $\begin{array}{l}85,2 \\
\%\end{array}$ & $76,8 \%$ & $91,0 \%$ \\
\hline Sensibilidad & $\begin{array}{l}76,5 \\
\%\end{array}$ & $62.2 \%$ & $86.8 \%$ \\
\hline \multirow{2}{*}{ Especificidad } & 93,0 & & \\
\hline & $\%$ & $82,2 \%$ & $97,7 \%$ \\
\hline \multirow{2}{*}{ Valor predictivo positivo } & 90,7 & & \\
\hline & $\%$ & $76,9 \%$ & $97,0 \%$ \\
\hline \multirow{2}{*}{ Valor predictivo negativo } & 81,5 & & \\
\hline & $\%$ & $69,6 \%$ & $89,7 \%$ \\
\hline
\end{tabular}

II: 65 pacientes, $V$-VI: 43 pacientes.

*Considera casos en $C D V+V I$ verdaderos positivos y casos en $C D I I$ verdaderos negativos.

\section{Discusión}

Los NT son cada vez más frecuentes y requieren evaluación citológica para descartar malignidad. La PAAF de NT guiada por ecografía es la herramienta más utilizada para estos fines $1,7,8,9,10$. Varios estudios han reportado una frecuencia de Bethesda I entre 2 al $16 \%$ y un riesgo de malignidad muy variable entre 2 al $32 \% \%^{8,13,15,16}$, lo que coincide con nuestro trabajo donde 
encontramos un $11 \%$ y $23,8 \%$ respectivamente. La frecuencia esta categoría no debe ser mayor al $10 \%{ }^{7}$. Su riesgo de malignidad se cree está sobreestimado, debido a que está establecida sobre un subgrupo de Bethesda I, los que fueron operados, y por tanto poseían alguna característica clínica o ecográfica que llevó a dicha conducta9. La categoría Bethesda II es definida como benigna, sin embargo, conlleva un riesgo de malignidad que oscila entre un 1 a $10 \%{ }^{1,8}$. Algunas series han reportado un mayor riesgo de malignidad a mayor tamaño tumoral, principalmente en aquellos mayores a $\geq 3-4 \mathrm{~cm}^{17,18}$. En nuestro estudio, el riesgo de malignidad para la categoría Bethesda II fue $18,5 \%$, elevado respecto a estudios publicados. Ante una PAAF Bethesda III la conducta podría ser re-punzar, realizar estudios de biología molecular o indicar tiroidectomía diagnóstica, si posee características clínicas o ecográficas de alta sospecha de malignidad ${ }^{1,9,10}$. La frecuencia estimada de Bethesda III es de $1-27 \%$ de todas las PAAF y su riesgo de malignidad oscila entre un 6 a $48 \%$ 1,8,9,13,19. Esta categoría debería ser utilizada como último recurso y su frecuencia por centro debería ser menor del $10 \%{ }^{7,9}$. En nuestra serie, encontramos un $10 \%$ de PAAF en dicha categoría con un riesgo de malignidad del $31,6 \%$, que coincide con lo reportado.

La categoría Bethesda IV conlleva un riesgo intermedio de malignidad, entre $15-30 \%{ }^{1,8}$. La conducta sugerida es quirúrgica, sin embargo, si se poseen pruebas moleculares pueden considerarse útiles ${ }^{1,9,10}$. Nuestro trabajo tiene una frecuencia de esta categoría de $23 \%$ con un riesgo de malignidad de $62,8 \%$, siendo éste elevado respecto al reportado.

En cuanto a la categoría Bethesda V y VI, tienen indicación quirúrgica. El riesgo de malignidad reportado es $50-75 \%$ y $97-99 \%$ respectivamente ${ }^{8,9}$. El riesgo de malignidad de la categoría Bethesda $\mathrm{V}$ en nuestra población es del $90,5 \%$, el cual coincide con los casos reportados. En nuestra serie sólo encontramos un paciente en la categoría Bethesda VI que resultó ser un carcinoma papilar de tiroides.

Está reportado que el SB posee buena sensibilidad y especificidad por lo cual es una herramienta recomendada para el diagnóstico de cáncer de tiroides ${ }^{1,3,8,19}$. En el trabajo publicado por MoraGuzmán I et al $^{19}$, se describe una sensibilidad del $98,9 \%$, especificidad del $84,4 \%$, VPP del $69,6 \%$ y un VPN del 99,5\% cuando de evalúa al SB como prueba de screening, es decir, aquella punción que indica una intervención quirúrgica. Bongiovanni $M$ et $\mathrm{al}^{8}$ describe una sensibilidad del $97 \%$, especificidad del 50,7\%, VPP del 55,9\% y VPN del $96,3 \%$. En nuestra población respecto a los parámetros de prueba diagnóstica del BS, al valorarlo como prueba de screening (análisis 1), se objetivó una sensibilidad del 84,6\%, especificidad del $72,6 \%$, VPP del 76,7\% y VPN del $81,5 \%$. Por lo tanto, nuestros resultados coinciden con la bibliografía publicada respecto a que el SB posee una buena exactitud diagnóstica que le permite formar parte de los métodos de screening de cáncer de tiroides.

Por otro lado, está descripto que los criterios citopatológicos del SB obtenidos por PAAF de NT para el diagnóstico de cáncer de tiroides tienen una buena capacidad para asegurar la existencia de malignidad $^{19}$. Con este propósito, se describe una sensibilidad del 98,6\%, especificidad del 97,6\%, VPP $93,5 \%$ y VPN del $99,5 \%{ }^{19}$. En nuestra población en el análisis 2 se objetivó una sensibilidad del 76,5\%, especificidad del 93,0\%, VPP del $90,7 \%$ y VPN del $81,5 \%$. La exactitud diagnóstica fue del $85,2 \%$. Estos datos, que coinciden con trabajos publicados previamente, permiten definir al BS como una herramienta fiable cuando se trata de confirmar la existencia de malignidad $^{8,19}$.

\section{Conclusión}

La exactitud diagnóstica de los criterios citopatológicos del Sistema Bethesda (SB) obtenidos por PAAF de nódulos tiroideos para el diagnóstico de cáncer de tiroides es buena, por lo cual es uno de los métodos recomendados en el algoritmo diagnóstico de cáncer de tiroides.

\section{Bibliografia}

1. Haugen B, Alexander E, Bible K et al. 2015 American Thyroid Association Management Guidelines for Adult Patients with Thyroid Nodules and Differentiated Thyroid Cancer: The American Thyroid Association Guidelines Task Force on Thyroid Nodules and Differentiated Thyroid Cancer. Thyroid. 2016;26(1):1-133.

2. Bueno F; Abelleira E; Smulever A et al. Respuesta parcial a lenvatinib como segunda línea de tratamiento en cáncer diferenciado avanzado de tiroides. Rev Arg Endocrinol Metab. 2018; 55(3):182-187.

3. Pitoia F, Califano I, Vázquez A et al. Consenso intersocietario sobre tratamiento y seguimiento de pacientes con cáncer diferenciado de tiroides. Rev Arg Endocrinol Metab 2014;51(2):85-118.

4. Franco Uliaque C, Pardo Berdún F, Laborda Herrero R et al. Utilidad de la ecografía en la evaluación de los nódulos tiroideos. Radiología. 2019; 58(5):380-388. 
5. Cabezón C. Carcinoma diferenciado de Tiroides. Segunda parte. Separata. 2014;22(4):1-36.

6. Pitoia F. Carcinoma diferenciado de Tiroides. Primera Parte. Separata. 2014; 22(2):1-36.

7. Cibas E, Ali S. The Bethesda System for Reporting Thyroid Cytopathology. Thyroid. 2009;19(11):1159-1165.

8. Bongiovanni M, Spitale A, Faquin W et al. The Bethesda System for Reporting Thyroid Cytopathology: A Meta-Analysis. Acta Cytologica. 2012;56(4):333-339.

9. Cibas E, Ali S. The 2017 Bethesda System for Reporting Thyroid Cytopathology. Journal of the American Society of Cytopathology. 2017;6(6):217-222.

10. Liu X, Medici M, Kwong $\mathrm{N}$ et al. Bethesda Categorization of Thyroid Nodule Cytology and Prediction of Thyroid Cancer Type and Prognosis. Thyroid. 2016;26(2):256-261.

11. Romero-Rojas A, Melo-Uribe M. Implementación del Sistema Bethesda para el informe de citología aspirativa de tiroides con seguimiento histopatológico: experiencia en un centro de tratamiento de cáncer. Revista Colombiana de Cancerología. 2014;18(1):3-7.

12. Mileva M, Stoilovska B, Jovanovska A et al. Thyroid cancer detection rate and associated risk factors in patients with thyroid nodules classified as Bethesda category III. Radiology and Oncology. 2018;52(4):370-376.

13. Cibas E, Baloch Z, Fellegara G et al. A Prospective Assessment Defining the Limitations of Thyroid Nodule Pathologic Evaluation. Annals of Internal Medicine. 2013;159(5):325.

14. Cameselle-Teijeiro J, Sobrinho-Simões M. Nueva clasificación de la OMS de los tumores tiroideos: una categorización pragmática de las neoplasias de la glándula tiroides. Endocrinología, Diabetes y Nutrición. 2018; 65(3):133-135.

15. Theoharis C, Schofield K, Hammers L et al. The Bethesda Thyroid Fine-Needle Aspiration Classification System: Year 1 at an Academic Institution. Thyroid. 2009;19(11):1215-1223.

16. Moon H, Kwak J, Choi $\mathrm{Y}$ et al. How to Manage Thyroid Nodules With Two Consecutive Non-Diagnostic Results on Ultrasonography-Guided Fine-Needle Aspiration. World Journal of Surgery. 2012;36(3):586-592.

17. Yip L. Thyroid Nodules $\geq 4 \mathrm{~cm}$ : Can Ultrasound and Cytology Reliably Exclude Cancer? Reply. World Journal of Surgery. 2014;38(6):1556-1557.

18. Wharry LI, McCoy KL, Stang MT et al. Thyroid nodules $(>/=4 \mathrm{~cm})$ : can ultrasound and cytology reliably exclude cancer? World J Surg. 2014, 38(3):614-621.

19. Mora-Guzmán I, Muñoz de Nova J, MarínCampos $\mathrm{C}$ et al. Rendimiento del sistema Bethesda en el diagnóstico citopatológico del nódulo tiroideo. Cirugía Española. 2018;96(6):363-368.

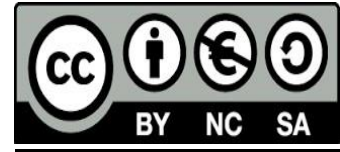

